\title{
Incidence, clinical features, and treatment of familial moyamoya in pediatric patients: a single-institution series
}

\author{
Jonathan Gaillard, BS, ${ }^{1}$ Jennifer Klein, NP, ${ }^{2}$ Daniel Duran, MD, ${ }^{1}$ Armide Storey, BS, ${ }^{2}$ \\ R. Michael Scott, MD, ${ }^{2}$ Kristopher Kahle, MD, PhD, ${ }^{1}$ and Edward R. Smith, MD ${ }^{2}$ \\ 'Department of Neurosurgery, Yale University, New Haven, Connecticut; and 'Department of Neurosurgery, Children's Hospital \\ Boston, Harvard Medical School, Boston, Massachusetts
}

OBJECTIVE Limited data exist on familial moyamoya in children. The purpose of this study was to characterize presentation and outcomes of pediatric moyamoya patients who have relatives diagnosed with moyamoya.

METHODS The authors performed a single-institution retrospective analysis of a case series including all surgically treated children with moyamoya with first- or second-degree relatives with moyamoya. Clinical and radiographic characteristics were analyzed, along with surgical outcomes.

RESULTS A total of 537 patients underwent surgery for moyamoya during the study period. Eighteen of those patients (3.4\% of the total series) had moyamoya and a family history of moyamoya and were included in this study. Of these 18 patients, 14 were non-twin siblings, and the remaining 4 represented 2 pairs of identical (affected) twins.

The presentation was predominantly ischemic $(72 \%)$, but 4 patients $(33 \%)$ were asymptomatic when they were found to have moyamoya. Bilateral disease was present in 13 patients (72\%). Radiographic stroke prevalence (67\%), Suzuki grade (3.3), and angiographic findings were comparable to findings in nonfamilial moyamoya patients. Thirty revascularization procedures were performed, with a 3.3\% operative stroke rate per hemisphere and no new strokes in an average follow-up period of 4.5 years.

CONCLUSIONS In a North American surgical series, familial moyamoya existed in $3.4 \%$ of cases, and was distinguished by manifesting in a broad range of ethnic groups, with a higher proportion of male patients and increased rates of asymptomatic and unilateral disease in comparison to nonfamilial moyamoya. Screening indications remain controversial and the current data are used to suggest guidelines. Surgical therapy is warranted, effective, and durable in these patients, but patients should be carefully selected.

https://thejns.org/doi/abs/10.3171/2016.12.PEDS16468

KEY WORDS moyamoya; familial; pediatric; stroke; pial synangiosis; screening; vascular disorders

$\mathrm{M}$ OYAMOYA is a progressive arteriopathy characterized by narrowing of the internal carotid arteries and their intracranial branches, resulting in compensatory collateral vessel development and increased risk of stroke. Initially described as an idiopathic process, there is now improved recognition and diagnosis of moyamoya associated with various genetic and systemic disorders, in addition to moyamoya-specific mutations. However, detailed study of the prevalence of familial risk of moyamoya remains limited in the pediatric population. Here we present our institutional experience, reviewing the clinical presentation, radiographic findings, and surgical treatment of a series of pediatric patients with moyamoya who also have other family members with moyamoya to better inform clinicians treating this population.

\section{Methods}

A consecutive surgical series of patients with moyamoya arteriopathy was reviewed to identify all patients 
with moyamoya and family members with moyamoya who were treated with revascularization between 1985 and 2015. Moyamoya was confirmed by the criteria outlined in the Japanese guidelines and recent pediatric evidence-based practice review. ${ }^{5,23}$ We defined "familial moyamoya" in accordance with these as a case involving a patient with moyamoya who also has at least 1 sibling, parent, aunt, or uncle with moyamoya.

In accordance with an institutional review board-approved protocol, medical records were retrospectively reviewed to determine patient demographic information, age at presentation and initial surgery, family history, symptoms at presentation, comorbid conditions, results of radiographic studies (including Suzuki grade), perioperative and late complications, and long-term outcomes. ${ }^{19,24}$ Clinical outcomes were assessed using the modified Rankin Scale (mRS) scores at presentation and at the 1-year follow-up visit, as previously described. ${ }^{19,20}$ Radiographic outcomes were assessed with Matsushima grade on the 1-year follow-up cerebral angiogram and also follow-up MRI data to identify any new strokes. ${ }^{13,28}$

In this series, 35 MRI with MR angiography (MRA) studies were performed on immediate siblings and relatives (18 patients and 17 first-degree relatives) either as part of evaluation due to symptoms (headache, transient ischemic attack [TIA], stroke) or as a screening examination because of an affected family member.

Of note, due to insurance and privacy issues, genetic testing is not done routinely nor made part of the patients' records at our institution. Consequently, we do not have comprehensive or uniform genetic analysis of the patients or family members in this group and therefore are not able to include these data in our study. We have initiated a multicenter research project to perform detailed, uniform sequencing of our patient (and family) cohort and hope to have results in the next year as a separate report.

\section{Results}

\section{Demographic Characteristics}

A total of 537 patients underwent surgery for moyamoya during the study period. Eighteen of those patients (3.4\% of the total series) had moyamoya and a family history of moyamoya and were included in this study. Of these 18 patients, 14 were siblings, and 4 represented 2 pairs of identical (affected) twins. The remaining patients had either a father or a maternal aunt who was affected. There were no affected first cousins reported.

Demographic information is summarized in Table 1, and family relationships are diagrammed in Fig. 1. There were 9 female and 9 male patients with moyamoya in this group. The average age at the time of treatment was 8.1 years (range 2-20 years). In contrast, the mean age at the time of treatment for the 519 patients with nonfamilial moyamoya was 7.1 years (range $0.5-21$ years). Two patients in the familial moyamoya cohort had sickle cell disease while the others had a negative medical history. Regarding ethnicity, 3 patients were of Asian ancestry (1 pair of siblings and 1 other patient), 4 were Eastern European Orthodox Jewish (2 pairs of siblings), 2 were African American (twin siblings, who also had sickle cell disease),
TABLE 1. Demographic characteristics of the patients included in this study

\begin{tabular}{cc}
\hline \multicolumn{1}{c}{ Characteristic } & Value \\
\hline No. of pts & 18 \\
\hline Sex & \\
\hline Male & 9 \\
\hline Female & 9 \\
\hline Age at 1st surgery (yrs) & \\
\hline Mean & 8.1 \\
\hline Range & $2-20$ \\
\hline
\end{tabular}

Pts = patients.

and the remainder were of Western European/Caucasian ancestry.

In 5 families, only 2 siblings existed, and all 10 of these children had MRI evidence of moyamoya. In 3 families, 1 parent was known to be symptomatic with moyamoya, so those 3 individuals were scanned, but the other parents (2 male, 1 female) were not scanned. Of note, we have a total of 7 sets of identical twins in our series of moyamoya patients. Of these 7 sets, 2 pairs are represented here, as both twins in each pair have moyamoya. In the other 5 sets, only 1 of the 2 identical twins has identifiable moyamoya (although 2 of these 5 sets may have early stage disease that does not yet meet formal defining criteria, but appear suspicious). Consequently, we can state that if 1 identical twin is found to have moyamoya, our series would indicate that the second twin has a $29 \%$ likelihood of having definitive moyamoya and a $29 \%$ likelihood of suspicious radiographic findings meriting follow-up.

\section{Clinical Presentation}

The average age at diagnosis was 8.1 years. Cerebral ischemia (including TIA or stroke) was the predominant presenting symptom (12 patients, 67\%), but 6 patients (33\%) were asymptomatic on presentation and were scanned as part of the screening process. See Table 2 for a complete summary of clinical presentation. A symptomatic stroke was present in 4 patients (22\%), TIAs occurred in 9 patients $(50 \%)$, seizures were documented in 2 patients (11\%), hemorrhage was documented in 1 patient $(6 \%)$, headaches were present in 7 patients (39\%), and 6 patients (33\%) were asymptomatic and diagnosed after MRI studies were performed as part of a familial screening process. Asymptomatic patients were offered surgical treatment in concordance with previously published criteria for surgical indications in asymptomatic moyamoya, including evidence of advanced arteriopathy (Suzuki Grade II-VI) and impaired cerebral blood flow (typically with a FLAIR ivy sign present in a distribution concordant with the moyamoya arteriopathy). ${ }^{14,23}$

\section{Radiographic Presentation}

There were 31 affected hemispheres in 18 patients (17 right, 16 left). Thirteen patients $(72 \%)$ had bilateral disease at presentation and 5 patients $(28 \%)$ had unilateral disease at presentation. Twelve patients (67\%) had radiographic evidence of stroke on imagining at presentation. The me- 

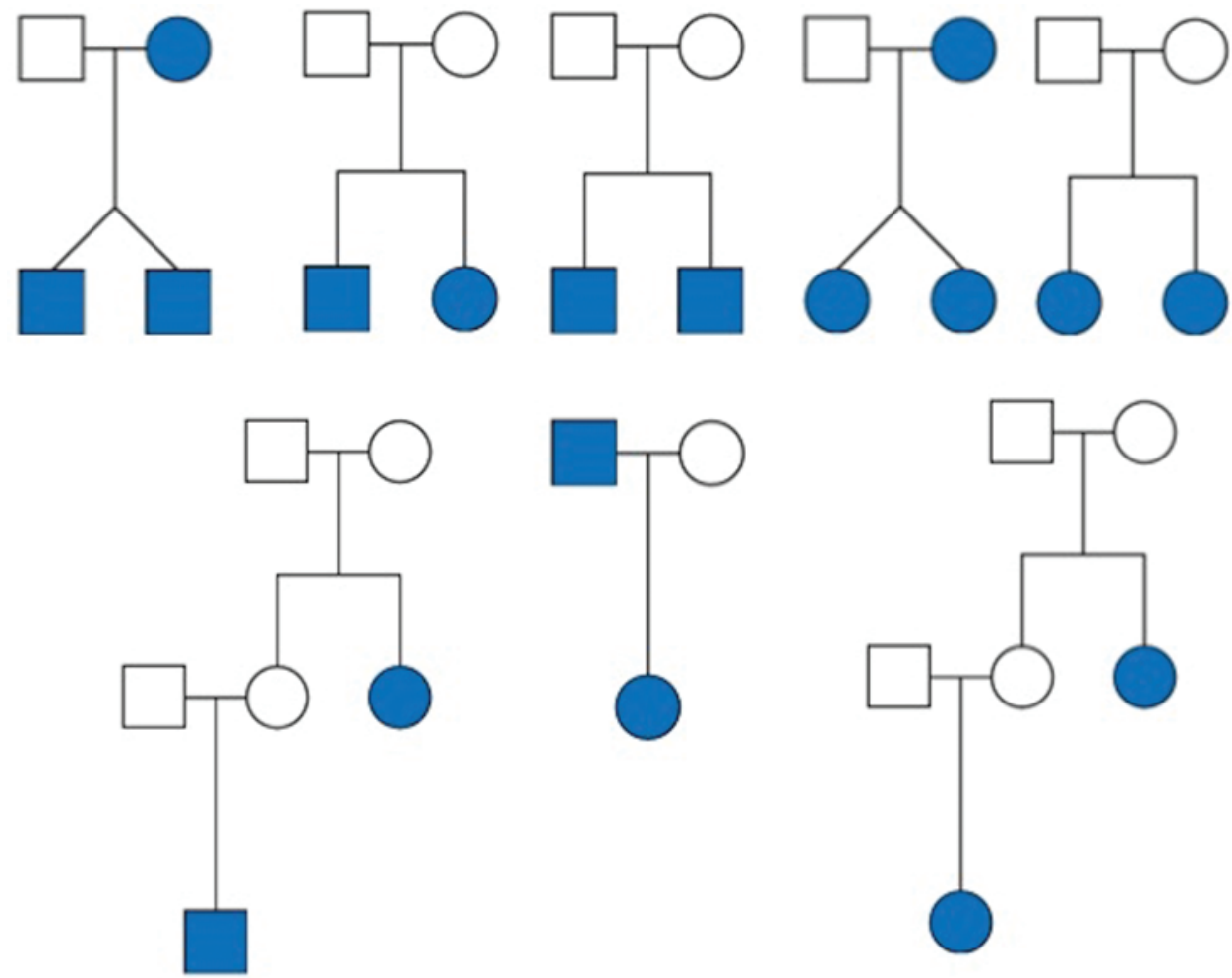

FIG. 1. The figure outlines the familial relationships of the patients in this study. A total of 18 individuals with moyamoya are included. Blue (gray in print) indicates individuals with moyamoya; white indicates unaffected individuals. Circles represent females; squares, males. An inverted "Y" denotes identical twins, while straight lines with right angles denote nonidentical siblings. Figure is available in color online only.

dian Suzuki grade was 3.3 for affected hemispheres $(\mathrm{Su}-$ zuki grade converted to Arabic for calculation). Seventeen patients (94\%) had FLAIR changes (ivy sign) on imaging (Table 3).

\section{Surgical Results}

A total 30 operations were performed in 18 patients at our institution (an additional operation was done on one side in a staged bilateral case prior to the patient's coming to our center-making the total caseload 31 cases in the

\section{TABLE 2. Clinical presentation}

\begin{tabular}{cc}
\hline \multicolumn{1}{c}{ Characteristic } & \multicolumn{1}{c}{ Value } \\
\hline Age at diagnosis (yrs) & \\
\hline Mean & 8.1 \\
\hline Range & $2-20$ \\
\hline Symptomatic at presentation & $12 / 18(67 \%)$ \\
\hline Clinical findings & $9 / 18(50 \%)$ \\
\hline TIA & $7 / 18(39 \%)$ \\
\hline Headache & $6 / 18(33 \%)$ \\
\hline Asymptomatic & $4 / 18(22 \%)$ \\
\hline Stroke & $2 / 18(11 \%)$ \\
\hline Seizure & $1 / 18(6 \%)$ \\
\hline Hemorrhage
\end{tabular}

Values are numbers of patients (\%) unless otherwise indicated.
18 patients; 30 at our institution and 1 elsewhere). Thirteen patients had bilateral procedures and 5 patients had unilateral procedures. Pial synangiosis was the primary operative technique in all 30 cases. Five of the patients undergoing bilateral surgeries had staged procedures, one of whom had one of his operations at an outside institution as mentioned above. (Although we now use a protocol in which moyamoya patients with bilateral disease are treated with both sides in a single anesthesia session, the 4 staged procedures from our institution reported in this study were performed at a time when the practice was to routinely stage bilateral operations.) The patients' average age at surgery was 8.1 years (range $2-20$ years).

\section{Complications}

In the 30 operations there were no deaths. Complica-

TABLE 3. Radiographic findings at presentation

\begin{tabular}{lc}
\hline \multicolumn{1}{c}{ Finding } & Value \\
\hline Radiographic stroke & $12 / 18(67 \%)$ \\
\hline Bilateral disease & $13 / 18(72 \%)$ \\
\hline Unilateral disease & $5 / 18(28 \%)$ \\
\hline Suzuki grade, median across 31 hemispheres & $3.3^{*}$ \\
\hline Ivy sign & $17 / 18(94 \%)$ \\
\hline Values are numbers of patients (\%) unless otherwise indicated. \\
* Suzuki grade has been converted to Arabic for calculation.
\end{tabular}


tions included 1 perioperative stroke (3.3\% stroke rate per hemisphere), which manifested as right arm and face weakness on postoperative Day 1, with a small infarction in the left middle cerebral artery distribution evident on imaging. At the 6-week follow-up visit, the child had recovered significantly, with only some face weakness remaining. One patient suffered a perioperative TIA, consisting of bilateral hand numbness and facial droop, which resolved within 24 hours without radiographic evidence of stroke (Table 4).

Of note, the 1 patient who was operated on at an outside institution had a perioperative stroke at that institution, which left the child with some cognitive delay and right arm weakness. These findings improved over a 6-month period.

\section{Outcomes}

Follow-up data were available for all patients with an average follow-up duration of 4.5 years (range 1-11.5 years). No other complications were noted beyond the perioperative stroke and TIA discussed previously. Comparison of preoperative and postoperative $\mathrm{mRS}$ scores showed stable or improved clinical status after surgery in all cases (preoperative mean 0.7 [range $0-3$ ] vs postoperative mean 0.4 [range 0-2]) (Table 4).

Postoperative MRI studies were available for review in 17 cases, and none showed evidence of new strokes. Postoperative angiograms were available for review in 12 cases, and all showed evidence of surgical collateral development. Of a total of 18 hemispheres, the Matsushima grade was $\mathrm{A}$ in $13(72 \%), \mathrm{B}$ in $2(11 \%)$, and $\mathrm{C}$ in $3(17 \%)$. Of the Grade C collaterals, 2 were from both sides of a single patient who underwent bilateral surgery and who had Suzuki Grade I and II disease preoperatively (the third was from a patient with Suzuki Grade IV unilateral disease).

\section{Discussion}

Moyamoya is a chronic cerebrovascular disorder initially described in Asia and now increasingly recognized elsewhere in the world. Recent studies have suggested that the moyamoya arteriopathy reported in North America and Europe has greater heterogeneity in clinical and radiographic presentation as compared with the original population described in Japan. ${ }^{1,8,18,21}$ The discovery of specific mutations associated with some forms of moyamoya suggest that there may be important genetic variation underlying these distinct populations.

While many groups-including our own-are performing ongoing investigations centered on genotyping moyamoya patients and families to better characterize the prevalence of specific mutations in populations, the data from those types of projects are distinct, but complementary, to the work presented here. As mentioned in our Methods section, due to insurance and privacy issues, genetic testing is neither done routinely nor made a part of the patient's records at our institution. The purpose of presenting the data in this report is not to describe prevalence of specific mutations, but rather to provide the clinician and affected families general information on the questions commonly asked about familial moyamoya in pediatric patients in North America.
TABLE 4. Clinical and radiographic outcomes

\begin{tabular}{cc}
\hline \multicolumn{1}{c}{ Variable } & Value \\
\hline Clinical follow-up in yrs & \\
\hline Average & 4.5 \\
\hline Range & $1-11.5$ \\
\hline Complications (periop) & 1 \\
\hline Stroke & \\
\hline TIA & 0.7 \\
\hline mRS score & $0-3$ \\
\hline Preop & \\
\hline Mean & 0.4 \\
\hline Range & $0-2$ \\
\hline Postop (1 yr) & \\
\hline Mean & \\
\hline Range & $13 / 18(72 \%)$ \\
\hline Matsushima grade at 1 yr postop & $2 / 18(11 \%)$ \\
\hline (18 hemispheres) & $3 / 18(17 \%)$ \\
\hline A &
\end{tabular}

These questions often include, "How often are other family members affected when a child is diagnosed with moyamoya?" "If a child has moyamoya, should we screen siblings for the disease?" and "Do children with familial moyamoya have different risks with surgery or different outcomes compared to nonfamilial cases?"

\section{What Are the Characteristics of Pediatric Familial Moyamoya Patients?}

In this North American surgical series, we examined pediatric patients with moyamoya disease who were treated at our institution between 1985 and 2015 and found that $3.4 \%$ of the cases $(n=18)$ were familial, and we report on those 18 cases here. This proportion is less than the previously reported proportion of 5.6\% (8 of 143 cases) that was identified in a study of cases treated between 1985 and 2001 at our institution..$^{19}$ This current study may prove a more accurate estimate of familial moyamoya disease in the United States due to the larger number of cases included (537 vs 143). Of note, not all aunts, uncles, and cousins were scanned, so it is possible that cases were undiagnosed in asymptomatic, unscanned family members. This means that our data may "undercall" the true prevalence of moyamoya, with a higher prevalence possible. Our practice has been to suggest scanning children once they are old enough to tolerate MRI without anesthesia (generally 5 years of age or older), to reduce risk of the study.

Interestingly, the proportion of moyamoya patients with familial moyamoya disease in this series is markedly lower than the proportions that have been found in Asian countries $(6 \%-15.4 \%$ in Japan, $9.4 \%$ in China, and $12 \%$ in Korea). ${ }^{2,3,9,11,26,29}$ It also is notably lower than recent estimates of $7.4 \%$ familial moyamoya in Western Europe. ${ }^{12}$ Regarding ethnicity, we had a very heterogeneous group of patients, with only 3 identifying Asian ancestry. (Of note, 
if we examine only patients of Asian ancestry within our group, the prevalence of familial cases within Asian patients is $5.5 \%$, higher than our group as a whole and closer-but still lower-than the reported rates from primary Asian countries.) The female-to-male ratio reported here is 1:1 for familial moyamoya, which differs markedly from the strong female preponderance reported for sporadic cases of moyamoya in the United States (with female-tomale ratios ranging from 1.6 to 2.8), Japan, and Korea (although similar to China's reported 1:1 ratio).3,7,9,25,27

\section{Do Familial and Sporadic Moyamoya Differ in Clinical or Radiographic Presentation?}

The age of onset in our series (8.1 years) was comparable to the ages of onset in Japan and China (7.2 and 8.0, respectively). ${ }^{3,17}$ As might be expected in a cohort that is often subjected to screening when other family members are found to have disease, one major difference in the presentation of familial moyamoya patients is the higher percentage of asymptomatic patients (33\% in this group compared with $15.5 \%$ in our total series and approximately $18 \%$, on average, from other nonfamilial series overseas). ${ }^{2,14}$ For patients who were symptomatic, however, the findings in familial and sporadic cases were similar, with both groups predominantly manifesting evidence of ischemic disease (stroke and/or TIA).

Radiographically, the findings of the familial moyamoya patients were very similar to those of the sporadic moyamoya patients. Specifically, the Suzuki grade and rates of evidence of radiographic stroke were nearly identical between groups. The one notable difference was the overall prevalence of unilateral disease in the familial group (28\%), which was double that in the sporadic cohort $(14 \%) .{ }^{22}$ Of the 5 patients with unilateral disease, 3 were identified as a result of family screening, suggesting that perhaps these patients might have been captured at an earlier point in their disease.

\section{How Does Treatment of Familial Moyamoya Compare With Treatment of Sporadic Disease?}

The risk involved in the surgical treatment of pediatric patients with familial moyamoya appears to be low, with a perioperative stroke rate of $3.3 \%$ per operated hemisphere, slightly lower than the $4 \%$ rate reported for the moyamoya population as a whole..$^{19}$ Long-term follow-up (mean 4.5 years) reveals that surgery confers durable protection from stroke in familial patients (with the annual stroke rate being essentially the same in both familial and nonfamilial moyamoya patients after revascularization), with improved mRS scores after operation and radiographic evidence of robust collateral growth, as evaluated by Matsushima grade.

Of note, 1 patient in our cohort had bilateral Matsushima " $C$ " collaterals, and this patient was also unique in having very early disease (Suzuki Grades I and II, left and right, preoperatively). This boy was asymptomatic and had no evidence of stroke on MRI, and his moyamoya was identified as a result of screening after moyamoya was diagnosed in his sister. While the disease met criteria for surgical treatment, it was less severe than many cases oper- ated on at our institution, and it is worth raising the discussion point - as a matter of speculation - that some patients with minimal disease may benefit from close observation rather than immediate operation, as there may be insufficient ischemia to foster surgical collateral growth., ${ }^{40} \mathrm{We}$ have previously reviewed specific indications for surgical intervention, which we have used in trying to determine treatment plans in cases of early-stage disease..$^{14,23}$

\section{Summary Points, Limitations, and Directions for Future Study}

This analysis of familial moyamoya in a pediatric population reveals data that stand in contrast to data from other reported series. Specifically, familial disease is substantially less common in this North American cohort (at $3.4 \%$ vs 2- to 5 -fold higher rates in other continents), affects a far broader spectrum of ethnic populations (beyond Asian), and affects males and females equally (as opposed to the female preponderance in sporadic cases). While the age of presentation is similar, patients with familial moyamoya are about twice as likely to be asymptomatic at presentation and also twice as likely to have unilateral disease, compared to those with nonfamilial moyamoya. Both of these findings may be reflective of the use of screening to detect early disease, and longer follow-up will better inform us as to how many of the cases of unilateral disease progress to bilateral disease.

In addition to the overall likelihood of finding moyamoya, 2 important questions remain to be answered. First, at what age should a child born into an affected family be screened by MRI? Our data reveal that most cases occurred after infancy, suggesting that it may be appropriate to wait until the child is older ( 5 years of age or older), allowing for safer imaging without general anesthesia. A second question centers on when (or if) repeated imaging is necessary in an unaffected sibling if an initial MRI shows no abnormality. Our current policy is to repeat imaging prior to independence (age 18-22 years), but this remains ad hoc, and our expectation is that following the cohort described in this paper will better inform subsequent practice.

Surgery with pial synangiosis is a low-risk, effective, and durable treatment for the prevention of stroke in the familial moyamoya patients, with long-term outcomes similar or superior to those reported in patients with sporadic disease. It is worth noting that the limited experience from our cohort of familial moyamoya patients may suggest a need to exercise restraint in offering surgery to patients with early-stage disease, as rates of progression in these early cases remain unclear.

Limitations to this study include the small number of patients, which we hope to increase through further patient accrual and the initiation of a multicenter study to pool data. As mentioned, another important, and complementary, data set for this work would be genotyping of all of the patients and family members, and we have already initiated a multicenter study to perform this analysis. Due to the length of time anticipated to complete the genetic analysis, coupled with the distinct and unique information provided by the analysis reported in this manuscript, we have elected to present this work now, with the genetic analysis to follow. 
Relevant to that work, our study here suggests an important aspect of future research, namely, the influence of environmental or other epigenetic factors on the manifestation of the moyamoya phenotype. ${ }^{6}$ Given that we have 7 sets of identical twins, with only 2 pairs fully expressing the arteriopathy phenotype (and 2 pairs with equivocal findings), there is compelling evidence that the moyamoya phenotype is variable in its penetrance and expression. Hypotheses to explain these phenomena have been forwarded, but definitive data remain elusive..$^{4,15-17}$ The identification of specific factors that regulate this arteriopathy may provide critical insights relevant to the development of novel therapeutics. ${ }^{6}$

A final question remains as to what findings might justify subjecting family members to screening if a child is found to have moyamoya. Currently, we define screening as MRI with MRA, and increasingly are adding referral to a genetic counselor (if possible) for known moyamoya mutations (specifically $R N F 213$ ). Based on the data presented here, our practice is to recommend that screening family members is not necessary in isolated, sporadic cases (given the low rate of 3.4\%) unless: 1) there are other known family members with moyamoya, 2) clinical history suggests symptoms strongly suggestive of moyamoya in other family members (TIA, stroke, severe headaches, or seizure), or 3) the patient is an identical twin. Given the higher prevalence in patients of Asian ancestry, we have a lower threshold to screen first-degree family members in this population. In general, we will recommend delaying MRI in very young children (under 1 year of age) due to the complexities of imaging in this age group and also typically recommend delaying screening other family members until successful completion of surgical treatment of the index patient (so as to reduce psychosocial burdens on the family). It remains unclear whether imaging should be repeated-or at what interval-should an initial screening MRI examination be negative.

\section{Conclusions}

Familial cases of pediatric moyamoya are less common in this North American cohort than in other series reported around the world, and are further distinguished by manifesting in a broad range of ethnic groups, with a higher ratio of males affected and increased rates of asymptomatic and unilateral disease. Surgical therapy is warranted, effective, and durable in these patients, but patients should be carefully selected. Screening remains a controversial topic, but the data presented here suggest general guidelines that can aid clinicians and families.

\section{Acknowledgments}

We would like to acknowledge the Marcus Chae Moyamoya Research Fund and the Kids at Heart Neurosurgery Research Fund.

\section{References}

1. Acker G, Goerdes S, Schneider UC, Schmiedek P, Czabanka M, Vajkoczy P: Distinct clinical and radiographic characteristics of moyamoya disease amongst European Caucasians.

Eur J Neurol 22:1012-1017, 2015

2. Baba T, Houkin K, Kuroda S: Novel epidemiological features of moyamoya disease. J Neurol Neurosurg Psychiatry 79:900-904, 2008

3. Bao XY, Duan L, Yang WZ, Li DS, Sun WJ, Zhang ZS, et al: Clinical features, surgical treatment, and long-term outcome in pediatric patients with moyamoya disease in China. Cerebrovasc Dis 39:75-81, 2015

4. Fujimura M, Sonobe S, Nishijima Y, Niizuma K, Sakata H, Kure S, et al: Genetics and biomarkers of moyamoya disease: significance of RNF213 as a susceptibility gene. J Stroke 16: $65-72,2014$

5. Fukui M: Guidelines for the diagnosis and treatment of spontaneous occlusion of the circle of Willis ('moyamoya' disease). Research Committee on Spontaneous Occlusion of the Circle of Willis (Moyamoya Disease) of the Ministry of Health and Welfare, Japan. Clin Neurol Neurosurg 99 (Suppl 2):S238-S240, 1997

6. Ganesan V, Smith ER: Moyamoya: defining current knowledge gaps. Dev Med Child Neurol 57:786-787, 2015

7. Graham JF, Matoba A: A survey of moyamoya disease in Hawaii. Clin Neurol Neurosurg 99 (Suppl 2):S31-S35, 1997

8. Hallemeier CL, Rich KM, Grubb RL Jr, Chicoine MR, Moran CJ, Cross DT III, et al: Clinical features and outcome in North American adults with moyamoya phenomenon. Stroke 37:1490-1496, 2006

9. Ikezaki K, Han DH, Kawano T, Kinukawa N, Fukui M: A clinical comparison of definite moyamoya disease between South Korea and Japan. Stroke 28:2513-2517, 1997

10. Ito A, Fujimura M, Niizuma K, Kanoke A, Sakata H, MoritaFujimura Y, et al: Enhanced post-ischemic angiogenesis in mice lacking RNF213; a susceptibility gene for moyamoya disease. Brain Res 1594:310-320, 2015

11. Kim SK, Cho BK, Phi JH, Lee JY, Chae JH, Kim KJ, et al: Pediatric moyamoya disease: an analysis of 410 consecutive cases. Ann Neurol 68:92-101, 2010

12. Kossorotoff M, Hervé D, Toulgoat F, Renaud C, Presles E, Chabriat H, et al: Paediatric moyamoya in mainland France: a comprehensive survey of academic neuropaediatric centres. Cerebrovasc Dis 33:76-79, 2012

13. Lin N, Aronson JP, Manjila S, Smith ER, Scott RM: Treatment of moyamoya disease in the adult population with pial synangiosis. J Neurosurg 120:612-617, 2014

14. Lin N, Baird L, Koss M, Kopecky KE, Gone E, Ullrich NJ, et al: Discovery of asymptomatic moyamoya arteriopathy in pediatric syndromic populations: radiographic and clinical progression. Neurosurg Focus 31(6):E6, 2011

15. Liu W, Morito D, Takashima S, Mineharu Y, Kobayashi H, Hitomi T, et al: Identification of RNF213 as a susceptibility gene for moyamoya disease and its possible role in vascular development. PLoS One 6:e22542, 2011

16. Mineharu Y, Liu W, Inoue K, Matsuura N, Inoue S, Takenaka $\mathrm{K}$, et al: Autosomal dominant moyamoya disease maps to chromosome 17q25.3. Neurology 70:2357-2363, 2008

17. Nanba R, Kuroda S, Tada M, Ishikawa T, Houkin K, Iwasaki Y: Clinical features of familial moyamoya disease. Childs Nerv Syst 22:258-262, 2006

18. Peerless SJ: Risk factors of moyamoya disease in Canada and the USA. Clin Neurol Neurosurg 99 (Suppl 2):S45-S48, 1997

19. Scott RM, Smith JL, Robertson RL, Madsen JR, Soriano SG, Rockoff MA: Long-term outcome in children with moyamoya syndrome after cranial revascularization by pial synangiosis. J Neurosurg 100 (2 Suppl Pediatrics):142-149, 2004

20. See AP, Ropper AE, Underberg DL, Robertson RL, Scott RM, Smith ER: Down syndrome and moyamoya: clinical presentation and surgical management. J Neurosurg Pediatr 16:58-63, 2015

21. Smith ER, Scott RM: Moyamoya: epidemiology, presentation, and diagnosis. Neurosurg Clin N Am 21:543-551, 2010 
22. Smith ER, Scott RM: Progression of disease in unilateral moyamoya syndrome. Neurosurg Focus 24(2):E17, 2008

23. Smith ER, Scott RM: Spontaneous occlusion of the circle of Willis in children: pediatric moyamoya summary with proposed evidence-based practice guidelines. A review. J Neurosurg Pediatr 9:353-360, 2012

24. Suzuki J, Takaku A: Cerebrovascular "moyamoya" disease. Disease showing abnormal net-like vessels in base of brain. Arch Neurol 20:288-299, 1969

25. Uchino K, Johnston SC, Becker KJ, Tirschwell DL: Moyamoya disease in Washington State and California. Neurology 65:956-958, 2005

26. Wakai K, Tamakoshi A, Ikezaki K, Fukui M, Kawamura T, Aoki R, et al: Epidemiological features of moyamoya disease in Japan: findings from a nationwide survey. Clin Neurol Neurosurg 99 (Suppl 2):S1-S5, 1997

27. Wetjen NM, Garell PC, Stence NV, Loftus CM: Moyamoya disease in the midwestern United States. Neurosurg Focus 5(5):e1, 1998

28. Yamada I, Matsushima Y, Suzuki S: Childhood moyamoya disease before and after encephalo-duro-arterio-synangiosis: an angiographic study. Neuroradiology 34:318-322, 1992

29. Yamauchi T, Tada M, Houkin K, Tanaka T, Nakamura Y, Kuroda $\mathrm{S}$, et al: Linkage of familial moyamoya disease (sponta- neous occlusion of the circle of Willis) to chromosome 17q25. Stroke 31:930-935, 2000

\section{Disclosures}

The authors report no conflict of interest concerning the materials or methods used in this study or the findings specified in this paper.

\section{Author Contributions}

Conception and design: Smith, Scott, Kahle. Acquisition of data: Gaillard, Klein, Duran, Storey, Kahle. Analysis and interpretation of data: Smith, Klein, Duran, Kahle. Drafting the article: Smith, Gaillard, Scott, Kahle. Critically revising the article: Smith, Scott. Reviewed submitted version of manuscript: Smith, Kahle. Approved the final version of the manuscript on behalf of all authors: Smith. Administrative/technical/material support: Gaillard, Klein, Duran, Storey. Study supervision: Scott.

\section{Correspondence}

Edward R. Smith, Department of Neurosurgery, Children's Hospital Boston, Harvard Medical School, 300 Longwood Ave., Boston, MA 02115.email: edward.smith@childrens.harvard.edu. 